\title{
Energy relaxation in hot electron quantum optics via acoustic and optical phonon emission
}

\author{
C. Emary, ${ }^{1}$ L. A. Clark, ${ }^{1}$ M. Kataoka, ${ }^{2}$ and N. Johnson ${ }^{3}$ \\ ${ }^{1}$ Joint Quantum Centre (JQC) Durham-Newcastle, School of Mathematics, Statistics and Physics, Newcastle University, \\ Newcastle-Upon-Tyne, NE1 7RU, United Kingdom \\ ${ }^{2}$ National Physical Laboratory, Hampton Road, Teddington, Middlesex TW11 0LW, United Kingdom \\ ${ }^{3}$ NTT Basic Research Laboratories, NTT Corporation, 3-1 Morinosato Wakamiya, Atsugi, Kanagawa 243-0198, Japan
}

(Received 31 July 2018; revised manuscript received 18 October 2018; published 22 January 2019)

\begin{abstract}
We study theoretically the relaxation of hot quantum-Hall edge-channel electrons under the emission of both acoustic and optical phonons. Aiming to model recent experiments with single-electron sources, we describe simulations that provide the distribution of electron energies and arrival times at a detector a fixed distance from the source. From these simulations we extract an effective rate of emission of optical phonons that contains contributions from both a direct emission process as well as one involving inter-edge-channel transitions that are driven by the sequential emission of first an acoustic and then an optical phonon. Furthermore, we consider the mean energy loss due to acoustic phonon emission and resultant broadening of the electron energy distribution and derive an effective drift-diffusion model for this process.
\end{abstract}

DOI: 10.1103/PhysRevB.99.045306

\section{INTRODUCTION}

Dynamical quantum dots have emerged as accurate, ondemand sources of single electrons [1-8]. One particularly novel feature of these charge pumps, as compared with other single-electron sources [9-13], is the high energy at which they inject electrons into quantum-Hall edge channels. This opens up the possibility of performing quantum-optics-like experiments with electrons [14-24] in a new energy regime. Clearly, the success of such experiments will depend critically on the relaxation and decoherence properties of the hot electrons as they are transmitted along edge channels. At low injection energies, electron-electron interactions will dominate the relaxation of hot electrons [25]. However, at high energies and fields, the transport electrons become ever more localized in the edge of the sample and their interaction with the cold, bulk electrons becomes much reduced. This then opens up the possibility that other relaxation mechanisms, principally phonon emission, will become the limiting inelastic mechanism.

Longitudinal-optical (LO) phonon emission from the hot electrons emitted by dynamic-quantum-dot single-electron sources has been observed in Refs. [4-8], and most recently studied in detail in Ref. [26]. The theory of direct LOphonon emission within a Fermi's golden rule approach was discussed in Ref. [27], the key prediction of which was that increasing magnetic field strength should dramatically suppress LO-phonon emission. In Ref. [26], however, rates of LO-phonon emission extracted from time-of-flight and survival-probability measurements were seen to be significantly greater than those predicted by this theory. The mechanism proposed to explain this was that the observed LO emission was, in fact, a sequential two-phonon process, where the electron first emits a longitudinal acoustic (LA) phonon and then the LO phonon.

In this paper we investigate more fully the role of acoustic phonons in the relaxation of hot single electrons and focus on LA phonons interacting via the deformation potential interaction, which is expected to be dominant in GaAs heterostructures [28]. We consider both LA and LO phonons and calculate the respective rates for emission by hot electrons in quantum-Hall edge channels. These rates then form the basis for the simulations of single electrons injected as localized wave packets into a quantum-Hall edge channel. These simulations allow us to calculate the energy-resolved arrival-time distribution (ATD) of the electrons at a detector some fixed distance from the electron source $[7,8]$. From this we extract survival probabilities, mean time-of-flight, and also the effective LO emission rate as in Ref. [26]. We study the energy and field dependence of this effective rate and highlight contributions from direct $\mathrm{LO}$ emission processes and the sequential LA+LO channel. We show how, in the regime dominated by the sequential channel, the effective LO rate is given by the total LA emission rate for inter-edge-channel scattering [29].

We then consider the impact of LA-phonon emission in its own right, and in particular, its influence on the distribution of electron energies observed at the detector. We show how this mechanism leads to a drop in mean energy of the electrons, as well as an increase in the width of their distribution in energy. Moreover, we show that these features can be captured by an analytically solvable drift-diffusion model and derive explicit expressions for the behavior of the energy cumulants. One key prediction of this model is that, at high energies, the width of electron energy distribution should be proportional to the square of the mean time of flight of the electrons. Furthermore, we show that the effects of acoustic phonons on the electron energy distribution increases algebraically with magnetic field.

With our focus on single-electron sources and observables pertinent to current experiments, our work differs considerably from earlier works on phonon emission in quantum-Hall systems, e.g., Refs. [29,30]. 
This paper is structured as follows. In Sec. II we set up our model of quantum-Hall electrons interacting with $\mathrm{LO}$ and LA phonons. In Sec. III we derive from this model phonon emission rates and write down a master equation. In Sec. IV we describe our simulations and the calculation of electron arrival-time distributions. Section V contains our discussion of the effective LO-phonon emission rate, and Sec. VI details our drift-diffusion model for relaxation under acoustic phonon emission. We finish with discussions in Sec. VII. A number of calculational details are given in the Appendix.

\section{ELECTRON-PHONON INTERACTIONS}

We consider a Hamiltonian $H=H_{\mathrm{e}}+H_{\mathrm{p}}+V_{\mathrm{ep}}$, where the three contributions respectively describe the electrons, the phonons, and electron-phonon interactions. The electrons we model as quasi-two-dimensional with strong confinement in the $z$ direction and weak harmonic confinement transverse to the transport direction. In the presence of perpendicular magnetic field strength $B$, electron states are described by the edge-channel index $n=0,1,2, \ldots$, and wave number $k>0$ in the transport direction [27,31]. The electron Hamiltonian reads

$$
H_{\mathrm{e}}=\sum_{n k} E_{n k} c_{n k}^{\dagger} c_{n k}
$$

with energies measured from the bottom of the $n=0$ subband,

$$
E_{n k}=n \hbar \Omega+\frac{1}{2}\left(\frac{\Omega}{\omega_{c}}\right)^{2} m_{e}^{*} \omega_{y}^{2} y_{\mathrm{G}}^{2}(k) .
$$

Here, $m_{e}^{*}$ is the effective electron mass, $\omega_{y}$ is the parabolic confinement frequency, $\Omega=\left(\omega_{y}^{2}+\omega_{c}^{2}\right)^{1 / 2}, \omega_{c}=|e B| / m_{e}^{*}$ is the cyclotron frequency, and

$$
y_{\mathrm{G}}(k)=\left(\frac{\omega_{c}}{\Omega}\right)^{2} \frac{\hbar k}{e B}
$$

is the "guide center" of the transverse wave function. The transverse extent of these wave functions is given by the length $l_{\Omega}=\left(\hbar / m_{e}^{*} \Omega\right)^{1 / 2}$, and the velocity of an electron with energy $E$ in edge channel $n$ is $v_{n}(E)=$ $\left(\omega_{y} / \Omega\right)\left[2(E-n \hbar \Omega) / m_{e}^{*}\right]^{1 / 2}[32]$.

We assume that the LO phonons are dispersionless with energy $\hbar \omega_{\mathrm{LO}}=36 \mathrm{meV}$ [25] and that the LA phonons have linear dispersion with speed of sound $c_{\mathrm{LA}}$. With annihilation operators $a_{\mathbf{q}}$ and $b_{\mathbf{q}}$ for LO and LA phonons of wave vector $\mathbf{q}$, the phonon Hamiltonian reads

$$
H_{\mathrm{p}}=\hbar \omega_{\mathrm{LO}} \sum_{\mathbf{q}} a_{\mathbf{q}}^{\dagger} a_{\mathbf{q}}+\hbar c_{\mathrm{LA}} \sum_{\mathbf{q}} q b_{\mathbf{q}}^{\dagger} b_{\mathbf{q}} .
$$

Specified in terms of $\tilde{c}_{\mathbf{k}}$, the annihilation operator for plane-wave electrons with three-dimensional wave vector $\mathbf{k}$, the Fröhlich Hamiltonian for electron-phonon interactions [33,34] reads

$$
\begin{aligned}
V_{\mathrm{ep}}= & \sum_{\mathbf{k}, \mathbf{q}} \mathcal{M}_{\mathrm{LO}}(\mathbf{q}) \tilde{c}_{\mathbf{k}+\mathbf{q}}^{\dagger} \tilde{c}_{\mathbf{k}}\left(a_{-\mathbf{q}}^{\dagger}+a_{\mathbf{q}}\right) \\
& +\sum_{\mathbf{k}, \mathbf{q}} \mathcal{M}_{\mathrm{LA}}(\mathbf{q}) \tilde{c}_{\mathbf{k}+\mathbf{q}}^{\dagger} \tilde{c}_{\mathbf{k}}\left(b_{-\mathbf{q}}^{\dagger}+b_{\mathbf{q}}\right) .
\end{aligned}
$$

The momentum dependence of the matrix elements is given by $[34,35]$

$$
\left|\mathcal{M}_{\mathrm{LO}}(\mathbf{q})\right|^{2} \equiv \frac{M_{\mathrm{LO}}^{2}}{L^{3}} \frac{1}{q^{2}}, \quad\left|\mathcal{M}_{\mathrm{LA}}(\mathbf{q})\right|^{2}=\frac{M_{\mathrm{LA}}^{2}}{L^{3}} q,
$$

where $q=|\mathbf{q}|$ and $L^{3}$ is the sample volume. Details of the couplings $M_{\mathrm{LO}}$ and $M_{\mathrm{LA}}$ are given in Appendix A. We have assumed here that the interaction with LA phonons is exclusively through the deformation-potential interaction (LADP for short) [36]. [We have also considered the effects of piezoelectric phonon interactions, but these are generally unimportant here (see Appendix D).]

In terms of the edge-channel states, the interaction reads

$$
\begin{aligned}
V_{\mathrm{ep}}= & \sum_{n n^{\prime}} \sum_{k k^{\prime}} \sum_{\mathbf{q}} \Lambda_{n^{\prime} k^{\prime} n k}^{\mathrm{LO}}(\mathbf{q}) c_{n^{\prime} k^{\prime}}^{\dagger} c_{n k}\left(a_{-\mathbf{q}}^{\dagger}+a_{\mathbf{q}}\right) \\
& +\sum_{n n^{\prime}} \sum_{k k^{\prime}} \sum_{\mathbf{q}} \Lambda_{n^{\prime} k^{\prime} n k}^{\mathrm{LA}}(\mathbf{q}) c_{n^{\prime} k^{\prime}}^{\dagger} c_{n k}\left(b_{-\mathbf{q}}^{\dagger}+b_{\mathbf{q}}\right),
\end{aligned}
$$

with matrix elements $\Lambda_{n^{\prime} k^{\prime} n k}^{\mathrm{LO}}$ and $\Lambda_{n^{\prime} k^{\prime} n k}^{\mathrm{LA}}$ obtained as in Ref. [27].

\section{RELAXATION RATES AND MASTER EQUATION}

We consider single electrons injected into the system, and since experiments are performed at low temperature, we consider phonon emission only. Let $P_{n k}$ be the probability to find an electron in edge channel $n$ with wave number $k$. Within a master equation approach [37], the equation for the evolution of these probabilities reads

$$
\dot{P}_{n k}=-\sum_{n^{\prime} k^{\prime} v} \Gamma_{n^{\prime} k^{\prime} n k}^{v} P_{n k}+\sum_{n^{\prime} k^{\prime} v} \Gamma_{n k n^{\prime} k^{\prime}}^{v} P_{n^{\prime} k^{\prime}},
$$

where $\Gamma_{n^{\prime} k^{\prime} n k}^{v}$ is the transition rate from state $n k$ to $n^{\prime} k^{\prime}$ induced by the emission of a $v=$ LO, LA phonon. According to Fermi's golden rule $[36,38]$, these rates read

$$
\Gamma_{n^{\prime} k^{\prime} n k}^{v}=\frac{2 \pi}{\hbar} \sum_{\mathbf{q}}\left|\Lambda_{n^{\prime} k^{\prime} n k}^{v}\right|^{2} \delta\left(E_{n^{\prime} k^{\prime}}-E_{n k}+\hbar \omega_{\nu}\right),
$$

with $\omega_{\nu}=\omega_{\mathrm{LO}}$ for $v=\mathrm{LO}$ and $\omega_{\nu}=\omega_{\mathrm{LA}}=c_{\mathrm{LA}} q$ for $v=$ LA. The explicit evaluation of these quantities for the LADP interaction is discussed in Appendix B. Details of the evaluation of the LO emission rates are as in Ref. [27].

Taking the continuum limit and defining the continuous distributions $\rho_{n}(E)=\sum_{k} \delta\left(E-E_{n k}\right) P_{n k}$ in terms of electron energy $E$, our master equation becomes

$$
\begin{aligned}
\dot{\rho}_{n}(E)= & -\sum_{n^{\prime} v} \int d E^{\prime} \widetilde{\Gamma}_{n^{\prime} n}^{v}\left(E^{\prime}, E\right) \rho_{n}(E) \\
& +\sum_{n^{\prime} v} \int d E^{\prime} \widetilde{\Gamma}_{n n^{\prime}}^{v}\left(E, E^{\prime}\right) \rho_{n^{\prime}}\left(E^{\prime}\right),
\end{aligned}
$$

with "rate densities"

$$
\begin{aligned}
\widetilde{\Gamma}_{n^{\prime} n}^{v}\left(E^{\prime}, E\right) \equiv & \frac{L}{2 \pi} \frac{1}{\hbar v_{0}\left(E^{\prime}\right)} \int d E_{k} \int d E_{k^{\prime}} \\
& \times \delta\left(E-E_{k}\right) \delta\left(E^{\prime}-E_{k^{\prime}}\right) \Gamma_{n^{\prime} k^{\prime} n k}^{v} .
\end{aligned}
$$




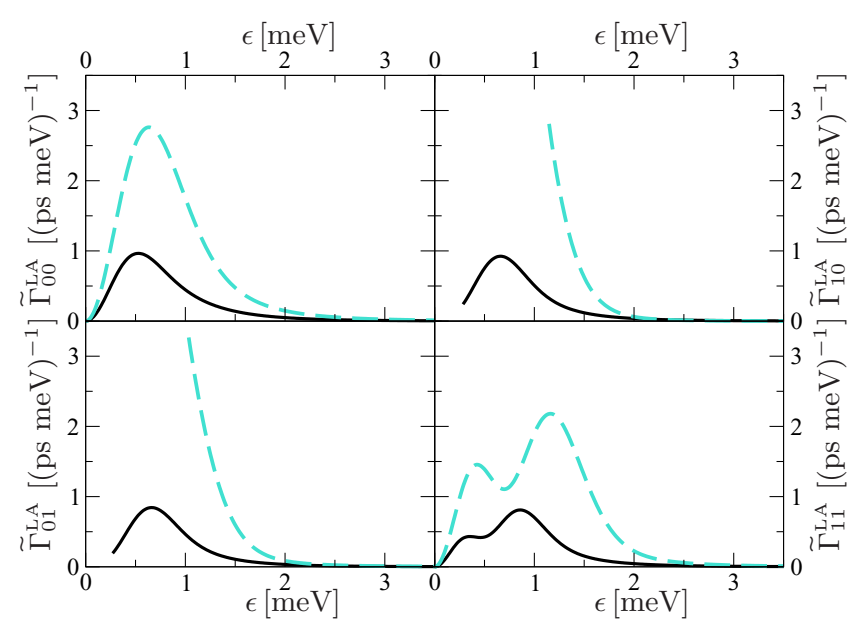

FIG. 1. The rate densities $\widetilde{\Gamma}_{n^{\prime} n}^{\mathrm{LA}}(E-\epsilon, E)$ as a function of energy loss $\epsilon$ for transitions induced by acoustic phonon emission in the outermost two edge channels $n, n^{\prime}=0,1$. The initial energy $E$ was $100 \mathrm{meV}$ above the $n=0$ band bottom, and results are shown for two values of the magnetic field: $B=6 \mathrm{~T}$ (black solid), $B=12 \mathrm{~T}$ (blue dashed). Other parameters are as set out in Appendix A. At these fields, the subband spacings are respectively $\hbar \Omega=10.3 \mathrm{meV}$ and $\hbar \Omega=20.9 \mathrm{meV}$. The rate densities are defined such that the area under each curve gives the total rate out of a state with energy $E$ and subband $n$ into subband $n^{\prime}$. The curves for off-diagonal rates end abruptly at low $\epsilon$ due to the cut-off Eq. (13).

The total scattering rate out of state in subband $n$ with energy $E$ into subband $n^{\prime}$ is given by the integral

$$
\Gamma_{n^{\prime} n}^{v \text { tot }}(E)=\int d E^{\prime} \widetilde{\Gamma}_{n^{\prime} n}^{v}\left(E^{\prime}, E\right) .
$$

Figure 1 shows these rate densities for transitions in the outermost two edge channels with magnetic field strengths of $B=6 \mathrm{~T}$ and $B=12 \mathrm{~T}$. For all transitions, we observe that LADP phonon scattering is significant only for changes in electron energy $\epsilon=E-E^{\prime}$ of a few meV. In addition, the rate densities at $6 \mathrm{~T}$ are smaller than their 12-T counterparts. Given the greater electron velocity at lower fields, most of this difference arises from the difference in density-of-states factor $1 /\left(\hbar v_{0}\right)$ in Eq. (11). The $n \neq n^{\prime}$ rates that describe interedge-channel scattering [29] are finite only above a certain minimum value of $\epsilon$ given by

$$
\epsilon \geqslant \hbar c_{\mathrm{LA}}\left|k-k^{\prime}\right| \text {. }
$$

This lower cutoff arises because of energy and momentum conservation and the fact that, when changing subband, a small drop in electron energy necessarily has associated with it a change in electron wave number.

\section{ARRIVAL-TIME DISTRIBUTION AND SIMULATIONS}

The stochastic nature of phonon emission means that different electrons emit a different sequence of phonons as they travel. This, coupled with the energy dependence of the electron velocity, means that after traveling a distance $x_{D}$ from source to detector, the electrons will arrive at a range of times. The distribution of such times is called the arrival-time distribution, and currently the most general such object accessible in experiment is the energy-resolved arrival-time distribution $A\left(E, \tau, x_{D}\right)$, defined such that $A\left(E, \tau, x_{D}\right) \Delta E \Delta \tau$ is the probability that an electron arrives at detector position $x_{D}$ with an energy between $E$ and $E+\Delta E$ between times $\tau$ and $\tau+\Delta \tau$ (in the limit $\Delta E, \Delta t \rightarrow 0$ ). The calculation of the ATD within quantum mechanics involves some subtleties [39-42]. However, we shall here pursue a semiclassical description [43] in which these issues do not arise.

We assume that the electrons are emitted in Gaussian wave packets [44] centered around an energy $E=E_{0}$ and time $t=0$, with energy and time widths of $\sigma_{E}=1 \mathrm{meV}$ and $\sigma_{\tau}=5 \mathrm{ps}$, in line with recent experiments $[7,8,45]$. Furthermore, we assume that these wave packets remain coherent during their transmission to the detector and that their shape remains constant. The absence of significant dispersion over the relevant timescales was discussed in [27]. The maintenance of coherence under phonon emission can be justified by considering the Bloch-Redfield equations for the coherences, analogous to those for the populations in Eq. (8). This analysis shows the rates for the transfer of coherences to be approximately the same as those for the transfer of populations [Eq. (9)] when the spread in $k$ vectors is $\lesssim l_{\Omega}^{-1}$. This is the case for the conditions studied here.

In this picture, then, phonon emission transfers traveling, fixed-shape wave packets between different energies. What remains is to track the motion of the center of these wave packets as they travel, and this we do using a Monte Carlo simulation of individual electron trajectories based on the master equation, Eq. (10).

We first discretize the electron energy, $E_{i}=i \Delta E ; i=$ $0,1,2, \ldots$. In discrete time step $\Delta t$, then, an electron in band $n$ with energy $E_{i}$ has a probability to emit a phonon and scatter into a new state with $E_{j}$ in band $n^{\prime}$ given by ${ }^{1}$

$$
T_{j i}^{n^{\prime} n} \approx \Delta t \int_{E_{j}-\Delta E / 2}^{E_{j}+\Delta E / 2} d E^{\prime} \widetilde{\Gamma}_{n^{\prime} n}\left(E^{\prime}, E_{i}\right) .
$$

During this time step, an electron will also propagate a distance $\Delta x=v_{n}\left(E_{i}\right) \Delta t$. Thus, our procedure is to iterate these two steps of probabilistic phonon emission and deterministic electron propagation until the distance traveled reaches $x_{D}$. This is then repeated many times and the convolution of this set of wave-packet centers in $E$ - $t$ space with the Gaussian wave packet of the individual electrons builds up a picture of the energy-resolved ATD.

For the results we present here, the electron always starts in the outermost edge channel, $n=0$ [44]. For numerical simplicity, our simulation considers only subbands $n=0,1$, where the majority of the dynamics takes place. We consider the detector to be positioned a distance $x_{D}=28 \mu \mathrm{m}$ from the center of the initial distribution.

Figures 2 and 3 show the energy-resolved ATD for two different starting energies $E_{0}=70,120 \mathrm{meV}$ with a field of $B=6 \mathrm{~T}$. Results are shown only for electrons detected

\footnotetext{
${ }^{1} \mathrm{We}$ found that numerical integration over the small energy interval is preferable to using the approximation $T_{j i}^{n^{\prime} n} \approx \Delta t \Delta E \widetilde{\Gamma}_{n^{\prime} n}\left(E_{j}, E_{i}\right)$, since this avoids problems with the cutoffs in Eq. (13), as well as with the rapid variations in the rates that occur in their vicinity.
} 


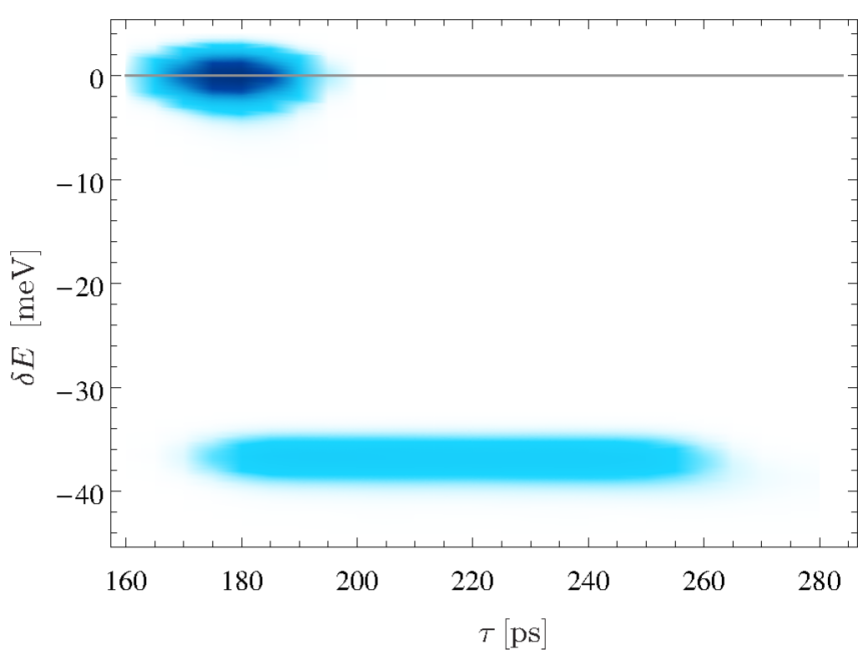

FIG. 2. Energy-resolved arrival-time distribution (ATD) $A\left(E, \tau, x_{D}\right)$ plotted as a function of energy loss $\delta E=E-E_{0}$, with $E_{0}$ the initial energy, and the arrival time $\tau$. White indicates absence of electrons, darker colors greater electron probability density. Clustered around $\delta E=0$ (horizontal line) is the ATD for electrons that arrive at the detector without having emitted any LO phonons. Around $\delta E=-\hbar \omega_{\mathrm{LO}}=-36 \mathrm{meV}$ we see the ATD of the first LO-phonon replica. Parameters: $B=6 \mathrm{~T}, E_{0}=70 \mathrm{meV}$, $x_{D}=28 \mu \mathrm{m}, \sigma_{E}=1 \mathrm{meV}$, and $\sigma_{\tau}=5 \mathrm{ps}$. The number of electron trajectories followed was $10^{5}$.

in the $n=0$ edge channel. This is by far the majority for these parameters, since only $<3 \%$ of electrons end up in the $n=1$ band at the detector. In both plots, a portion of the electron density remains clustered around the original injection energy $\delta E=E-E_{0}=0$ with central arrival time of $\tau=176 \mathrm{ps}$ for $E_{0}=70 \mathrm{meV}$ (Fig. 2) and $\tau=136 \mathrm{ps}$ for $E_{0}=120 \mathrm{meV}$ (Fig. 3). These distributions represent those electrons that reach the detector without having emitted any LO phonons, and this part of the ATD we shall denote as the $A^{(0)}$ distribution.

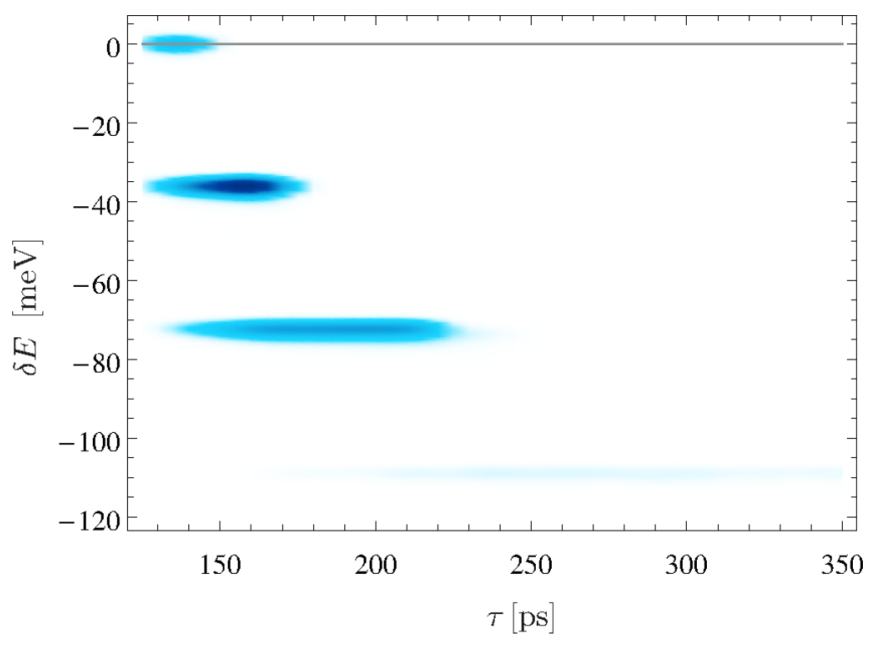

FIG. 3. As in Fig. 2, but here with the higher injection energy of $E_{0}=120 \mathrm{meV}$. Distributions corresponding to three LO-phonon replicas (the third is faint), plus that of the directly transmitted electrons, are observed.
The ATD also shows clusters positioned approximately around energy losses equal to multiples of the LO-phonon energy. These are the LO-phonon replicas, in which electrons have emitted $1,2, \ldots$ LO phonons en route to the detector. In Fig. 2 one replica is visible; in Fig. 3 there are three, and this number depends on both the initial energy as well as the distance of travel. The initial energy sets the maximum number of phonon replicas that can be observed, since once within $\hbar \omega_{L O}$ of the band bottom, no further LO phonons can be emitted. The distance traveled determines the degree to which each of these phonon replicas are actually realized, with lower-energy replicas becoming more populated the further the electron travels. As is clear from these figures, the phonon-replica distributions arrive later than the original distribution due to the energy lost to LO phonons. They are also broadened along the time axis, which is due to the uncertainty in the emission time of the LO phonons and corresponding uncertainty in the fraction of total distance traveled in lower-energy states.

\section{EFFECTIVE LO-PHONON EMISSION RATE}

The LO-phonon rate analysis of Ref. [26] was based on estimation of the "survival probability," i.e., the probability of reaching the detector without having emitted a LO phonon or, in other words, the total weight of the $A^{(0)}$ distribution. Although a counting-field approach would permit the calculation of exactly that part of the distribution having emitted $m$ LO phonons, we elect here instead to use a procedure based on inference from the ATD that matches the experimental procedure. With only LO-phonon processes active, it is a simple matter to infer this probability from the energy distribution of the electrons at the detector-surviving electrons reach the detector with exactly the same energy with which they are injected. In the presence of the continuous energy loss from LA-phonon emission, however, this picture is complicated by the fact that the electron energy distributions drop and broaden as they transit. Therefore we here define "survival" to mean that the energy of the electron at the detector satisfies $E>$ $E_{\text {surv }}=E_{0}-\hbar \omega_{L O}+10 \mathrm{meV}$. The choice of $10 \mathrm{meV}$ here is somewhat arbitrary, but the value should be large enough to ensure we avoid the tails of the emitted distribution and yet small enough that the $A^{(0)}$ distribution does not drop near this line under the emission of acoustic phonons. The value of $10 \mathrm{meV}$ was found to perform well in both these respects. In terms of the ATD, the survival probabilities are obtained as

$$
P_{n}=\int d E \int d \tau A_{n}\left(E, \tau, x_{D}\right) \Theta\left(E-E_{\text {surv }}\right),
$$

where $P_{n}$ and $A_{n}$ are quantities conditioned on finding the electrons in edge channel $n$. Thus, $P_{0}$ is the survival probability with the electron being detected in the outermost edge channel; $P_{1}$ is the same but with the electron being detected in the $n=1$ channel.

Figure 4(a) shows survival probabilities $P_{0,1}$ as a function of injection energy for $B=6,12 \mathrm{~T}$. At low energies, $P_{0} \approx 1$, with the vast majority of electrons reaching the detector without having emitted an LO phonon and being detected in the original edge channel. As injection energy increases above $E_{0}>\hbar \omega_{\mathrm{LO}}$, however, $P_{0}$ drops rapidly as LO emission becomes active. The other striking feature in Fig. 4(a) is that, 


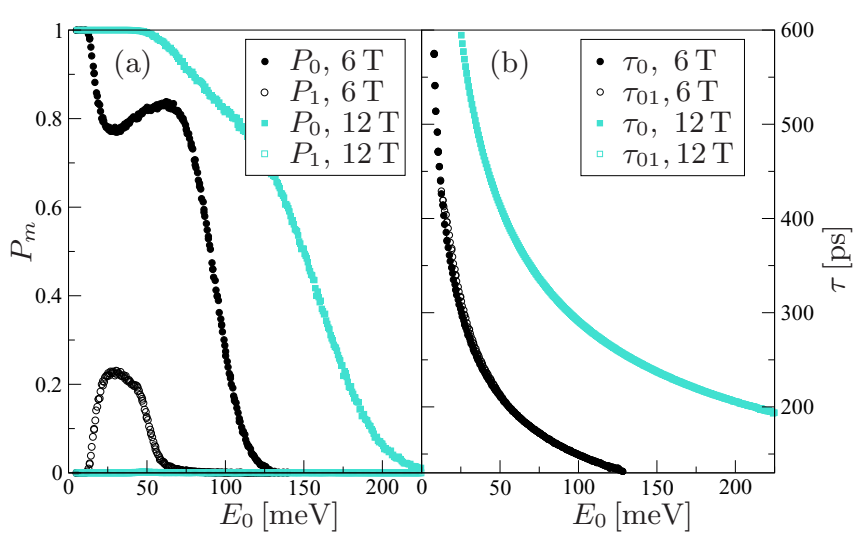

FIG. 4. (a) The survival probabilities $P_{0}$ and $P_{1}$ as a function of injection energy $E_{0}$ for two magnetic field strengths, $B=6,12 \mathrm{~T}$. (b) Mean time of flight for electrons in the $A^{(0)}$ distribution, i.e., those that reach the detector without having emitted a LO phonon. $\langle\tau\rangle_{0}$ counts only those electrons detected in the outermost edge channel, whereas $\langle\tau\rangle_{01}$ counts those in both $n=0,1$ states. The difference between these two quantities is seen to be minor.

at low field, we see that there exists an energy range for which $P_{1}$ is significant (up to about $22 \%$ in the figure). For higher fields, as exemplified by $B=12 \mathrm{~T}$, however, this feature is almost completely absent.

If LO-phonon emission was the only process operative here, then the survival probability $P_{0}$ would show an exponential decay as a function of time with rate given by the LO-phonon emission rate [27]. Let us therefore assume that a similar relation exists in the presence of LA-phonon emission and write an exponential relation between the survival probability, $P_{0} \approx e^{-\gamma_{0} t}$, where $\gamma_{0}$ is an effective rate parameter describing the total decay of the survival probability. We then approximate the time $t$ in this expression with $\langle\tau\rangle_{0}$ the mean time of flight for electrons in the $A^{(0)}$ distribution and outermost edge channel [this is shown in Fig. 4(b)]. Thus, the expression we use to extract the effective $\mathrm{LO}$ emission rate is

$$
\gamma_{0}=-\langle\tau\rangle_{0}^{-1} \log P_{0} .
$$

We also define $\gamma_{01}=-\langle\tau\rangle_{01}^{-1} \log \left(P_{0}+P_{1}\right)$, which takes into account "survived" electrons in both $n=0$ and $n=1$ subbands.

Simulation results for $\gamma_{0}$ and $\gamma_{01}$ are shown in Fig. 5 for both $B=6 \mathrm{~T}$ and $B=12 \mathrm{~T}$. At high energies, $\gamma_{0}$ and $\gamma_{01}$ are similar and both very well approximated by the bare LO-phonon emission rate (green solid lines). In this regime, therefore, we expect the conclusions of Ref. [27] to hold. At lower energies, however, the effective rates develop a "knee," which is more pronounced at lower fields and also more extreme in $\gamma_{0}$ than $\gamma_{01}$. Responsible for this enhancement is a relaxation process that involves first the emission of an LA phonon from the outermost edge channel to the $n=1$ channel, followed by the sequential emission of an LO phonon from $n=1$ back to the outermost edge channel. This process is best first analyzed by looking at the $B=12 \mathrm{~T}$ case, Fig. 5(b). Alongside simulation results $\left(\gamma_{0}\right.$ and $\gamma_{01}$ are pretty much identical at this field), we plot the direct LO rates from $n=0$ to $n^{\prime}=0$ (solid green lines) and from $n=1$

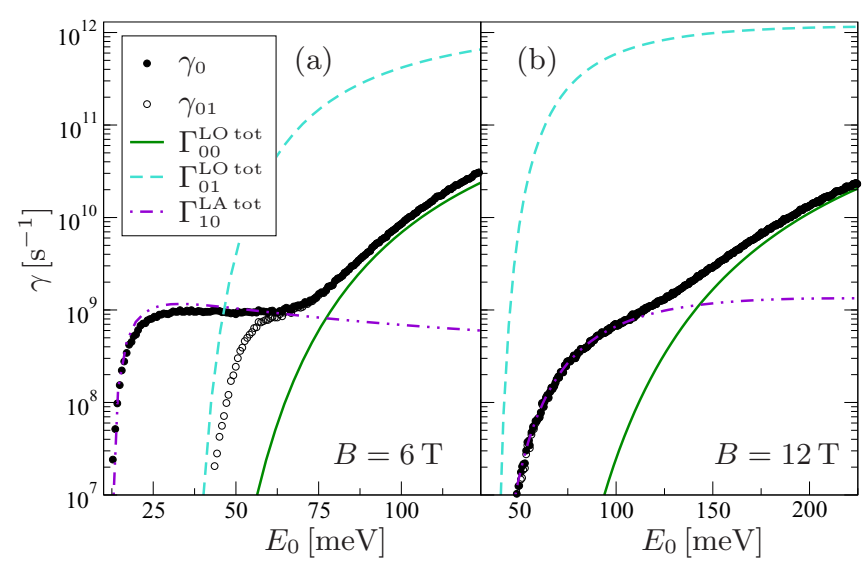

FIG. 5. The extracted LO emission rates $\gamma_{0}$ (solid circles) and $\gamma_{01}$ (empty circles) as a function of injection energy for (a) $B=6 \mathrm{~T}$ and (b) $B=12 \mathrm{~T}$. Also plotted are the calculated LO emission rates $\Gamma_{00}^{\mathrm{LO} \text { tot }}, \Gamma_{01}^{\mathrm{LO} \text { tot }}$ and total LA emission rate $\Gamma_{10}^{\mathrm{LA} \text { tot }}$.

to $n^{\prime}=0$ (dashed blue lines). We also plot the total LA rate from $n=0$ to $n^{\prime}=1$ (dot-dashed purple lines). At high energy, $\Gamma_{00}^{\mathrm{LO} \text { tot }} \gg \Gamma_{10}^{\mathrm{LA} \text { tot }}$ and the direct optical phonon process dominates. Then, as the energy decreases there is a crossover

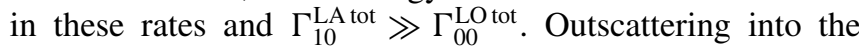
$n=1$ edge via LA emission then dominates over direct LO emission. However, the rate $\Gamma_{01}^{\mathrm{LO} \text { tot }}$ from $n=1$ back to $n=0$ is yet greater than all other rates considered and thus, upon arriving in the $n=1$ subband the electron rapidly emits an LO phonon and relaxes back into the $n=0$ subband. The speed of this two-step process is governed by the slowest step, which is the LA-phonon emission. Thus, in the "knee" region, the effective LO rate $\gamma_{0}$ is well approximated by the total LA rate $\Gamma_{10}^{\mathrm{LA}}$ tot. This approximation is observed to work well in Fig. 5(b). Furthermore, the rapidity of the $n=1$ to $n=0 \mathrm{LO}$ process also explains why for $B=12 \mathrm{~T}$ the population of the $n=1$ level at the detector is always small.

The story for $B=6 \mathrm{~T}$ is similar [Fig. 5(a)], with one significant difference. The rate $\Gamma_{01}^{\mathrm{LO} \text { tot }}$ drops rapidly, and around an energy of $E_{0} \approx 47 \mathrm{meV}$, falls below that of $\Gamma_{10}^{\mathrm{LA} \text { tot }}$. Below this point, there is no longer a rapid outscattering of electrons back from the $n=1$ to $n=0$ level. This then leads to the finite occupancy of the $n=1$ level which, in Fig. 4, is observed from $E_{0} \approx 47 \mathrm{meV}$ down until the rates drop off near the band bottom. This reduced outscattering also means that there is a significant difference between the effective rates $\gamma_{0}$ and $\gamma_{01}$ in this regime.

\section{ACOUSTIC-PHONON-INDUCED DRIFT DIFFUSION IN ENERGY SPACE}

Emission of LA phonons leads electrons to lose energy and, unlike $\mathrm{LO}$ emission, this occurs over a continuous range of energies. Furthermore, the stochasticity of this process leads to a broadening of the electron distributions as they travel. This broadening is just about apparent in Fig. 2, where the standard deviations of the $A^{(0)}$ distributions are greater than the starting value of $1 \mathrm{meV}$; the shift in the center of distribution is too slight to see from this figure for these parameters. 
As in the last section, we here focus on the behavior of the $A^{(0)}$ electrons and will write down an approximate analytic theory for the behavior of this distribution. To do so, we first discard LO processes (these we assume can be taken into account by an overall loss factor $e^{-\gamma_{0} t}$ which, since $\gamma_{0}$ is effectively constant over the energy scale relevant for LA emission, can simply be removed by normalizing the $A^{(0)}$ distribution) and confine ourselves to the outermost $n=0$ edge channel. To simplify notation, we thus drop the LA superscript and channel index for this section.

With injection energy much higher than the scale of any loss due to LA-phonon emission, the dispersion relation can be linearized

$$
E_{n k} \approx \varepsilon_{n}^{(0)}+\hbar v_{0} k
$$

where $v_{0}$ is the electron velocity, here assumed constant, and $\varepsilon_{n}^{(0)}$ is the energy offset. As shown in Appendix C, this linearization means that the LA-phonon rate becomes dependent only on the energy difference and we thus write $\widetilde{\Gamma}\left(E^{\prime}, E\right) \rightarrow \widetilde{\Gamma}\left(E-E^{\prime}\right)$ for $E>E^{\prime}$. The relevant part of our master equation, Eq. (10), then becomes

$$
\dot{\rho}(E)=\int_{0}^{\infty} d \epsilon \widetilde{\Gamma}(\epsilon)[-\rho(E)+\rho(E+\epsilon)],
$$

where we have extended the limits of integration commensurate with the linearization.

Equation (18) can be solved by introducing the generating function of energy moments

$$
\rho(\chi, t) \equiv \int_{-\infty}^{\infty} d E e^{i \chi E} \rho(E, t)
$$

We then obtain

$$
\dot{\rho}(\chi, t)=[\Lambda(\chi)-\Lambda(0)] \rho(\chi, t)
$$

with

$$
\Lambda(\chi) \equiv \int_{0}^{\infty} d \epsilon \widetilde{\Gamma}(\epsilon) e^{-i \chi \epsilon}
$$

Equation (20) can be solved to give

$$
\rho(\chi, t)=e^{[\Lambda(\chi)-\Lambda(0)] t} \rho(\chi, 0),
$$

such that the cumulant-generating function for the system is

$$
\mathcal{F}(\chi, t) \equiv \ln \rho(\chi, t)=[\Lambda(\chi)-\Lambda(0)] t+\mathcal{F}(\chi, 0),
$$

where $\mathcal{F}(\chi, 0)$ describes the energy statistics of the initial distribution. From this we obtain the $k$ th cumulant (subscript c) as

$$
\left\langle E^{k}(t)\right\rangle_{c}=\left.\frac{\partial^{k}}{\partial(i \chi)^{k}} \mathcal{F}(\chi, t)\right|_{\chi=0} .
$$

Explicit expressions for the first two cumulants read

$$
\langle E(t)\rangle=v_{E} t+E_{0}, \quad\left\langle E^{2}(t)\right\rangle_{c}=2 D_{E} t+\sigma_{E}^{2},
$$

where $E_{0}=\langle E(0)\rangle, \sigma_{E}^{2}=\left\langle E^{2}(0)\right\rangle_{c}$, and

$$
v_{E} \equiv-\int_{0}^{\infty} d \epsilon \epsilon \widetilde{\Gamma}(\epsilon), \quad D_{E} \equiv \frac{1}{2} \int_{0}^{\infty} d \epsilon \epsilon^{2} \widetilde{\Gamma}(\epsilon) .
$$

These last two quantities are the mean drift velocity of the electron energy distribution and the diffusion constant [46] associated with the spreading of the distribution. The higher cumulants of the $A^{(0)}$ distribution are nonzero (and could be calculated straightforwardly within this approach). Indeed, a distribution starting as a Gaussian leaves a small tail at higher energies as it relaxes downwards. For relevant parameters, this tail is small and approximation of the complete behavior as a Gaussian with just the first two cumulants provides a reasonably accurate description of the entire distribution.

The integrals of Eq. (26) can be computed numerically. They can also be evaluated approximately using a saddlepoint technique as outlined in Appendix C. This approximation yields the expressions

$$
\begin{aligned}
& v_{E} \approx-\frac{1}{16 \sqrt{2 \pi}} \frac{c_{\mathrm{LA}} M_{\mathrm{LA}}^{2}}{a^{3} l_{\Omega} \hbar v_{0}}, \\
& D_{E} \approx \frac{1}{4(2 \pi)^{3 / 2}} \frac{c_{\mathrm{LA}}^{2} M_{\mathrm{LA}}^{2}}{a^{4} l_{\Omega} v_{0}} .
\end{aligned}
$$

The derivation of these expressions also uses the fact that the ratio $c_{\mathrm{LA}} / v_{0}$ is small. Physically, this means that the phonons are emitted preferentially in a direction transverse to the electron propagation. Figures 6(a) and 6(b) show both full and approximate values for $v_{E}$ and $D_{E}$. Here we have plotted $\widetilde{v}_{E} \equiv v_{E} / v_{0}$ and $\widetilde{D}_{E} \equiv 2 D_{E} / v_{0}$, as these give the energy drop and variance increase per unit distance traveled. We see that, except for close to the band bottom, the energy lost is a fraction of a meV per micron. The diffusion constant gives a similar increase in variance per micron. These figures also show that our approximations capture the qualitative behavior well across the energy range but are quantitatively most accurate in the high-energy limit.

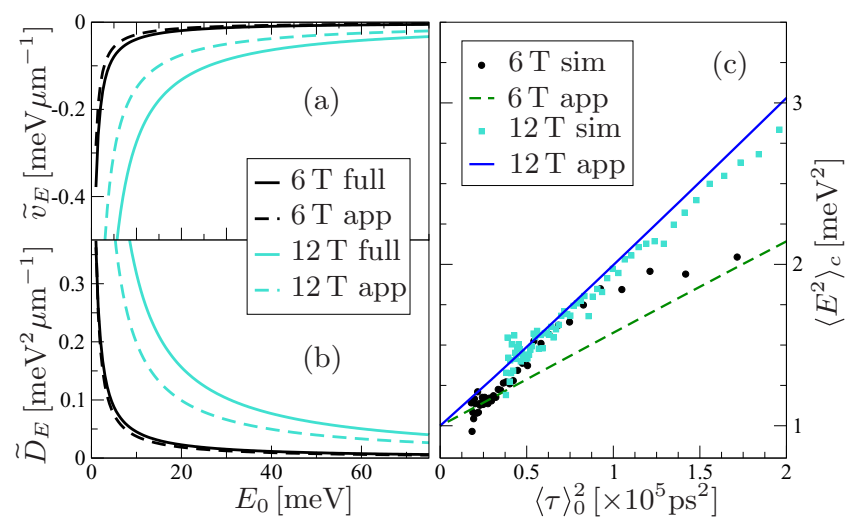

FIG. 6. Drift diffusion of the $A^{(0)}$ electron distribution. (a) The scaled energy-drift parameter $\widetilde{v}_{E} \equiv v_{E} / v_{0}$, which gives the mean energy loss of the electron per distance traveled (in $\mathrm{meV} / \mu \mathrm{m}$ ). (b) The similarly scaled diffusion parameter $\widetilde{D}_{E} \equiv 2 D_{E} / v_{0}$, which gives how much the variance increases per distance traveled (in $\mathrm{meV}^{2} / \mu \mathrm{m}$ ). Solid lines are from the full integrals of Eq. (26), whereas the dashed lines show the approximate forms from Eqs. (27) and (28). (c) The variance $\left\langle E^{2}\right\rangle_{c}$ of the $A^{(0)}$ distribution as a function of mean time-of-flight-squared $\langle\tau\rangle_{0}^{2}$ for $B=6,12 \mathrm{~T}$. The symbols represent results extracted from simulations; the straight lines are the analytic result from Eq. (29) with coefficients calculated from the full integrals. 
The only energy dependence in Eqs. (27) and (28) is through the $1 / v_{0}$ factor. Thus, with time of flight $\tau=x_{D} / v_{0}$ we can rewrite Eq. (25) as

$$
\langle E(t)\rangle=\lambda_{1} \tau^{2}+E_{0}, \quad\left\langle E^{2}(t)\right\rangle_{c}=\lambda_{2} \tau^{2}+\sigma_{E}^{2},
$$

where

$$
\lambda_{1}=\frac{v_{E} v_{0}}{x_{D}}, \quad \lambda_{2}=\frac{2 D_{E} v_{0}}{x_{D}}
$$

are energy-independent gradients. Thus plots of the energy drop and variance against the mean time of flight for the $A^{(0)}$ distribution should be straight lines. Figure 6(c) illustrates this for the energy variance. To compare with simulations, we extract the $A^{(0)}$ distribution in the outer edge channel and renormalize with the survival probability $P_{0}$. From this we extract the variance of the distribution and the mean time of flight. In the high-energy range shown here (corresponding to low $\tau^{2}$ ), the analytic theory matches the simulations well at high field (here $B=12 \mathrm{~T}$ ). Deviations from the analytic result for $B=6 \mathrm{~T}$ are apparent, but this is not surprising, given that at lower fields we have significant scattering out of the $n=0$ edge channel. We might expect further improvements in the agreement at even higher energies. However, here the survival probability becomes very small and statistics from simulations becomes unreliable (as would also be the case in experiment). At lower energies (high $\tau^{2}$ ), we come close to the band bottom, and the approximations used here cease to be valid.

\section{DISCUSSION}

From the point of view of probing fundamental semiconductor physics, single-electron sources offer rich possibilities. The results here show that the information encoded in the single-electron ATD can give significant insight into acoustic and optical phonon emission processes. Nevertheless, for applications, and particularly applications in the arena of hot electron quantum optics, it would presumably be best if all inelastic processes could be "switched off." The results we have presented here show that there are significant experimental handles that can be used to modify and suppress both LO and LA interactions. However, many of these handles effect the strength of the two interactions in opposite ways and thus care must be taken to find an optimal tradeoff between the two.

At low injection energies, $E_{0}<\hbar \omega_{\mathrm{LO}}$, the lack of possible destination states means that LO-phonon emission is absent. However, in this regime, the effects of LA-phonon emission are most pronounced, mainly because of the low velocity of electrons. ${ }^{2}$ At higher energies, the relaxation effects of LA phonons are considerably suppressed, mainly due the speed of transit of the electrons through the system. However, higher energy means an increase in activity of LO-phonon emission.

\footnotetext{
${ }^{2}$ The electron velocity is important on two accounts: first, faster electrons obviously have less time in the system during which to emit phonons; second, the phonon emission rates depend inversely on the electron velocity thanks to the one-dimensional density of electronic states entering in Eq. (11).
}

The role of magnetic field strength also plays a conflicting role. While increasing $B$ suppresses the LO emission rate exponentially, it increases the effects of LA emission, and quantities such as mean energy drop and energy spread increase algebraically with magnetic field. Thus it would appear that intermediate values of injection energy and magnetic field offer a good compromise.

More significantly, perhaps, is the dependence of the acoustic phonon emission rates on the strength of confinement in the $z$ direction, which enters our calculation through a parameter $a$ that characterizes the extent of wave function in this direction [see Eq. (A3)]. The energy lost to phonons scales like $\langle\Delta E\rangle \sim a^{-3}$, and the distribution width scales like $\sqrt{\left\langle E^{2}\right\rangle_{c}} \sim a^{-2}$. $^{3}$ This means that the most prominent effects of acoustic phonon emission can be significantly reduced by using a wider well (and hence larger $a$ ) to define the two-dimensional electron gas. From Ref. [27] we see that the LO-phonon rates are, to first approximation, independent of this width and should thus be unaffected by this.

In the calculations presented here, we have considered only electron-acoustic-phonon scattering via the deformation potential interaction. In GaAs, piezoelectric field scattering is also known to be significant in low-dimensional geometries in some parameter regimes [35]. We have repeated our simulations with these piezoelectric interactions present, and as we show in Appendix D, these generally have little influence. The only exception to this is at low fields in the region where a significant population of the $n=1$ subband is observed at the detector. This population is increased slightly by the piezoelectric interactions.

We have focused here exclusively on the case where electrons are injected into the outermost edge channel, corresponding to the situation generally held to be the case in experiments with dynamic-quantum-dot sources $[7,8,45]$. Injection into the $n=1$ might one day be possible, and we here comment on this possibility. On the numerical side, it is clear that our approach can be extended to this scenario (e.g., Fig. 1 contains the $n=1$ to $n^{\prime}=1$ acoustic phonon rate). Within the analytic approach of Sec. VI, both drift and diffusion parameters in the $n=1$ subband are scaled by a factor of $1 / \sqrt{3}$ relative to those within the $n=0$ subband. However, the most significant effect of injecting into the $n=1$ subband is that the fast LO emission process $n=1 \rightarrow n^{\prime}=0$ involved in the two-phonon emission described in Sec. V is now a relaxation channel directly open to the hot electron, rather than needing first the emission of an LA phonon. For this reason we expect the LO emission rates from the inner channels to be significantly increased over those for the outermost channel; see Ref. [27] for more details.

The Monte Carlo procedure that we have used to find the arrival-time distribution is manifestly a semiclassical one, where the rates are determined by quantum mechanics but the behavior of the electron distribution is taken to be a fixed wave packet around a classical trajectory. As discussed in Ref. [27], this approach is justified for time-of-flight-type experiments with hot electrons, e.g., [26], where the effects of

\footnotetext{
${ }^{3} \mathrm{~A}$ general energy cumulant scales like $\left\langle E^{k}\right\rangle_{c} \sim 1 / a^{k+2}$.
} 
quantum dispersion can largely be neglected. However, in future quantum-optics style experiments relying on interference, this approach will need to be extended to treat coherences as well as populations. It also remains as future work to evaluate the extent to which residual interactions with the Fermi-sea electrons contribute to the relaxation and decoherence of these electrons. In this context, we note that much progress has been made in understanding the decoherence and relaxation of low-energy single-electron sources through bosonization techniques, e.g., Refs. [47-49].

\section{ACKNOWLEDGMENTS}

We are grateful to H.-S. Sim and A. Dyson for useful discussions. This research was supported by EPSRC Grant No. EP/P034012/1. M.K. was supported by the UK Department for Business, Energy, and Industrial Strategy, and by EMPIR project 17FUN04 SEQUOIA. This project has received funding from the EMPIR program, cofinanced by the Participating States, and from the European Union's Horizon 2020 Research and Innovation Program.

\section{APPENDIX A: ELECTRON-PHONON MATRIX ELEMENTS AND CONFINEMENT PARAMETERS}

We take the matrix element for the interaction of electrons with LO phonons to be [34]

$$
M_{\mathrm{LO}}^{2}=4 \pi \alpha \hbar \frac{\left(\hbar \omega_{\mathrm{LO}}\right)^{3 / 2}}{\left(2 m_{e}^{*}\right)^{1 / 2}},
$$

with the following parameters for GaAs: electron effective mass, $m_{e}^{*}=0.067 m_{e}$; LO-phonon energy, $\hbar \omega_{\mathrm{LO}}=36 \mathrm{meV}$; and coupling constant, $\alpha=0.068$.

For the interaction with acoustic phonons, we assume the LADP interactions with matrix element [35]

$$
M_{\mathrm{LA}}^{2}=\frac{\hbar D^{2}}{2 d c_{\mathrm{LA}}},
$$

with density $d=5310 \mathrm{~kg} \mathrm{~m}^{-3}$, crystal acoustic deformation potential $D=8.6 \mathrm{eV}$, and speed of sound $c_{\mathrm{LA}}=4720 \mathrm{~ms}^{-1}$.

In contrast to Ref. [27], which considered a square-well confinement in the $z$ direction, we consider here a triangular quantum well with ground-state wave function given by the Fang-Howard ansatz [50],

$$
\phi_{0}(z)=\left(2 a^{3}\right)^{-1 / 2} z e^{-z /(2 a)}
$$

where the parameter $a$ determines the extent of the wave function. Here we choose a value of $a=3 \mathrm{~nm}$. For the transverse harmonic confinement, we take a parameter of $\hbar \omega_{y}=2.78$ $\mathrm{meV}$, obtained as a fit to the $V_{G 5}=-0.25 \mathrm{~V}$ time-of-flight results of Ref. [26].

\section{APPENDIX B: EVALUATION OF LADP EMISSION RATES}

Analogous to the LO-phonon calculation of Ref. [27], the rates of Eq. (9) for LADP emission can be written

$$
\begin{aligned}
\Gamma_{n^{\prime} k^{\prime} n k}^{\mathrm{LA}}= & \frac{2 \pi}{\hbar} \sum_{\mathbf{q}}\left|\mathcal{M}_{\mathrm{LA}}(\mathbf{q})\right|^{2}\left|G^{(z)}\left(q_{z}\right)\right|^{2}\left|G_{n^{\prime} k^{\prime}, n k}^{(y)}\left(q_{y}\right)\right|^{2} \\
& \times \delta_{q_{x}, k^{\prime}-k} \delta\left(E_{n^{\prime} k^{\prime}}-E_{n k}+\hbar c_{\mathrm{LA}} q\right)
\end{aligned}
$$

with

$$
\left|G_{n^{\prime} k^{\prime}, n k}^{(y)}\left(q_{y}\right)\right|^{2}=F_{n^{\prime} n}^{(y)}\left(\sqrt{\frac{1}{2} l_{\Omega}^{2}\left[q_{y}^{2}+\left(\frac{\omega_{c}}{\Omega}\right)^{2}\left(k^{\prime}-k\right)^{2}\right]}\right)
$$

and

$$
F_{n^{\prime} n}^{(y)}(Q)=\frac{n_{<} !}{n_{>} !} e^{-Q^{2}} Q^{2\left|n^{\prime}-n\right|}\left[L_{n_{<}}^{\left|n^{\prime}-n\right|}\left(Q^{2}\right)\right]^{2} .
$$

We consider here a triangular quantum well in the $z$ direction, for which the structure factor reads

$$
\left|G^{(z)}\left(q_{z}\right)\right|^{2}=F^{(z)}\left(a q_{z}\right) ; \quad F^{(z)}(Q)=\frac{1}{\left(1+Q^{2}\right)^{3}} .
$$

To evaluate Eq. (B1), we take the continuum limit and switch to polar coordinates: $q_{x}=|q| \cos \theta ; q_{y}=|q| \sin \theta \cos \phi ; q_{z}=$ $|q| \sin \theta \sin \phi$. Writing

$$
q_{0}=\left(E_{n k}-E_{n^{\prime} k^{\prime}}\right) / \hbar c_{\mathrm{LA}}
$$

and

$$
\cos \theta_{0}=\left(k^{\prime}-k\right) / q_{0}, \quad \theta_{0} \in[\pi / 2, \pi],
$$

we reduce Eq. (B1) to

$$
\begin{aligned}
\Gamma_{n^{\prime} k^{\prime} n k}^{\mathrm{LA}}= & \frac{1}{2 \pi \hbar^{2} c_{\mathrm{LA}} L} \int_{0}^{2 \pi} d \phi q_{0}\left|L^{3 / 2} \mathcal{M}_{\mathrm{LA}}\left(q_{0}, \theta_{0}, \phi\right)\right|^{2} \\
& \times F^{(z)}\left(a q_{0} \sin \theta_{0} \sin \phi\right)\left|G_{n^{\prime} k^{\prime} n k}^{(y)}\left(q_{0} \sin \theta_{0} \cos \phi\right)\right|^{2} \\
& \times \Theta\left(E_{n k}-E_{n^{\prime} k^{\prime}}-\hbar c_{\mathrm{LA}}\left|k^{\prime}-k\right|\right) \\
& \times \Theta\left(E_{n^{\prime} k^{\prime}}-n^{\prime} \hbar \Omega\right) .
\end{aligned}
$$

The first unit-step function $\Theta$ here arises from conservation of energy and momentum [see Eq. (13)], and the second avoids $E^{\prime}$ coming in lower than the band bottom.

\section{APPENDIX C: ESTIMATION OF DIFFUSION PARAMETERS}

The linearization of Eq. (17) means that for $n=n^{\prime}=0$ we have $\cos \theta_{0}=-c_{\mathrm{LA}} / v_{0}$, which is constant in this approximation, and also that the $\left(k-k^{\prime}\right)^{2}$ in the argument of $G_{n^{\prime} k^{\prime} n k}^{(y)}$ becomes $\left(E-E^{\prime}\right)^{2} /\left(\hbar v_{0}\right)^{2}$. Thus we see that the expression for the rate depends only on the difference $\epsilon=E-E^{\prime}$.

Introducing the energy scale $E_{z}=\hbar c_{\mathrm{LA}} / a$, the diffusion parameter in this approximation becomes

$$
D_{E} \approx \frac{M_{\mathrm{LA}}^{2}}{4 \pi \hbar^{3} a^{2} c_{\mathrm{LA}} v_{0}} E_{z}^{3} I_{D}
$$

with the integral

$$
\begin{aligned}
I_{D} \equiv & \frac{1}{\pi} \int_{0}^{\infty} d \tilde{\epsilon} \int_{-\pi / 2}^{\pi / 2} d \phi \tilde{\epsilon}^{4} \exp \left[-\frac{1}{2} r_{V}^{2} \tilde{\epsilon}^{2}\right] \\
& \times F^{(z)}\left(\tilde{\epsilon} \sin \theta_{0} \cos \phi\right) \exp \left[-\frac{1}{2} r_{A}^{2} \tilde{\epsilon}^{2} \sin \theta_{0} \sin ^{2} \phi\right]
\end{aligned}
$$

Here $\tilde{\epsilon} \equiv\left(E-E^{\prime}\right) / E_{z}$ is the dimensionless energy loss, and we have defined the ratios

$$
r_{V}=\frac{\omega_{c} c_{\mathrm{LA}} l_{\Omega}}{\Omega v_{0} a} \quad \text { and } \quad r_{A}=\frac{l_{\Omega}}{a}
$$


To obtain an approximate analytic form for this integral we first note that, for typical parameters, $r_{V} \sim 1 / 50$ compared with $r_{A} \sim 1 / 2$. We thus drop the initial exponential factor. We then approximate the integral over $\phi$ with a saddle-point approximation about $\phi=\pi / 2$. This gives

$$
I_{D} \approx \sqrt{\frac{2}{\pi}} \frac{1}{r_{A}} \frac{1}{\sin ^{5} \theta_{0}} \int_{0}^{\infty} d u u^{3} F^{(z)}(u)
$$

For the triangular well, this last integral can be performed analytically and we obtain

$$
I_{D} \approx \frac{1}{2 \sqrt{2 \pi} r_{A} \sin ^{5} \theta_{0}} .
$$

Finally, since for typical injection energies $c_{\mathrm{LA}} / v_{0} \ll 1$, we can approximate $\sin \theta_{0} \approx 1+\mathcal{O}\left(c_{\mathrm{LA}}^{2} / v_{0}^{2}\right)$, such that we obtain Eq. (28). A similar calculation for the energy-drift-velocity yields Eq. (27).

\section{APPENDIX D: LAPZ AND TAPZ INTERACTIONS}

The two additional acoustic phonon scattering mechanisms in GaAs are electron scattering due to the piezoelectric field by longitudinal phonons (LAPZ interaction) and transverse phonons (TAPZ interaction). These can be incorporated in our calculations with additional interaction terms in the Hamiltonian similar in structure to the acoustic phonon terms in Eq. (5) with matrix elements [35]

$$
\left|M_{\mathrm{LAPZ}}(\mathbf{q})\right|^{2}=\frac{32 \pi^{2} \hbar e^{2} h_{14}^{2}}{\varepsilon_{r}^{2} d c_{\mathrm{LA}} L^{3}} \frac{\left(3 q_{x} q_{y} q_{z}\right)^{2}}{q^{7}}
$$

and

$$
\begin{aligned}
\left|M_{\mathrm{TAPZ}}(\mathbf{q})\right|^{2}= & \frac{32 \pi^{2} \hbar e^{2} h_{14}^{2}}{\varepsilon_{r}^{2} d c_{\mathrm{TA}} L^{3}} \\
& \times\left|\frac{q_{x}^{2} q_{y}^{2}+q_{y}^{2} q_{z}^{2}+q_{z}^{2} q_{x}^{2}}{q^{5}}-\frac{\left(3 q_{x} q_{y} q_{z}\right)^{2}}{q^{7}}\right| .
\end{aligned}
$$

For parameter values we take $c_{\mathrm{TA}}=3340 \mathrm{~ms}^{-1}, \varepsilon_{r}=12.9$, and $h_{14}=1.41 \times 10^{9} \mathrm{~V} \mathrm{~m}^{-1}$.

Figure 7 shows the rate densities for these interactions in comparison with those for the LADP interaction for $B=$ $12 \mathrm{~T}$. For the interchannel scattering (determinant for the $\mathrm{LA}+\mathrm{LO}$ knee in the effective rate), the LAPZ and TAPZ rates are clearly much smaller than the LADP rate. For the intrachannel case, the situation is a bit more complicated. The LAPZ interaction is clearly negligible compared with LADP. For most of the range of energy loss $\epsilon$, the same is true for the TAPZ interaction. However, for $\epsilon \rightarrow 0$, the TAPZ rate density tends to a constant value, whereas the LADP density falls to zero. That this excess of the TAPZ rate density at small energy changes is generally unimportant can be appreciated by considering the cumulant integrals such as in Eq. (26).

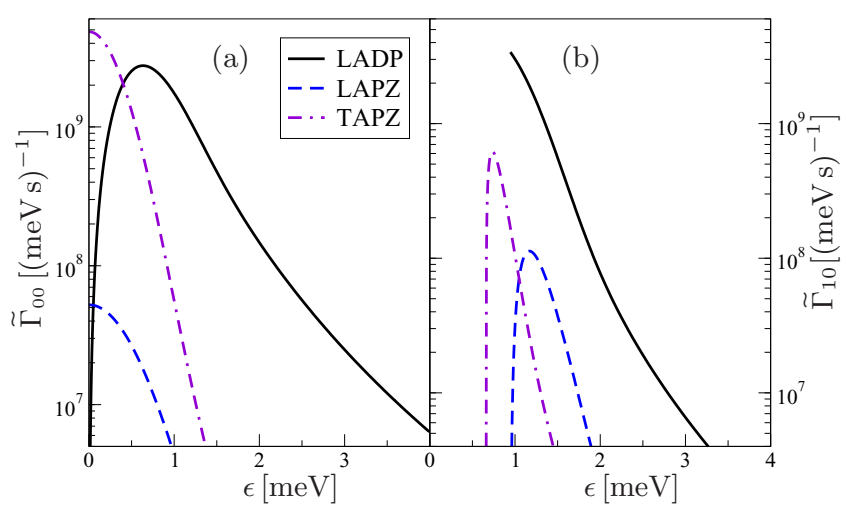

FIG. 7. Phonon emission rate densities $\widetilde{\Gamma}_{n^{\prime} n}(E-\epsilon, E)$ as a function of energy loss $\epsilon$ for the three acoustic phonon interactions: LADP (solid black lines), LAPZ (blue dashed), and TAPZ (violet dashed-dot). Left: transitions within the outermost LL $n=0 \rightarrow n^{\prime}=$ 0 . Right: first inward transition $n=0 \rightarrow n^{\prime}=1$. The magnetic field was $B=12 \mathrm{~T}$, and the starting energy was $E=100 \mathrm{meV}$.

Since the integrand for the $k$ th cumulant involves a factor $\epsilon^{k+1}$ [see Eq. (C3)], the magnitude of the rate at $\epsilon \rightarrow 0$ does not contribute to any cumulant, and hence the behavior of the entire energy distribution. The influence of the two piezoelectric interactions on the results presented here can be appraised from Fig. 8, which shows the survival probabilities $P_{0,1}$ calculated both with and without them. For $B=12 \mathrm{~T}$, the piezoelectric terms make no appreciable difference.

At lower field $(B=6 \mathrm{~T})$ the strength of the two piezoelectric interactions relative to the LADP interaction increases. The overall effect of this is again small, except for the region where we have significant population of the $m=1$ level. Here the piezoelectric processes lead to a small increase in this population.

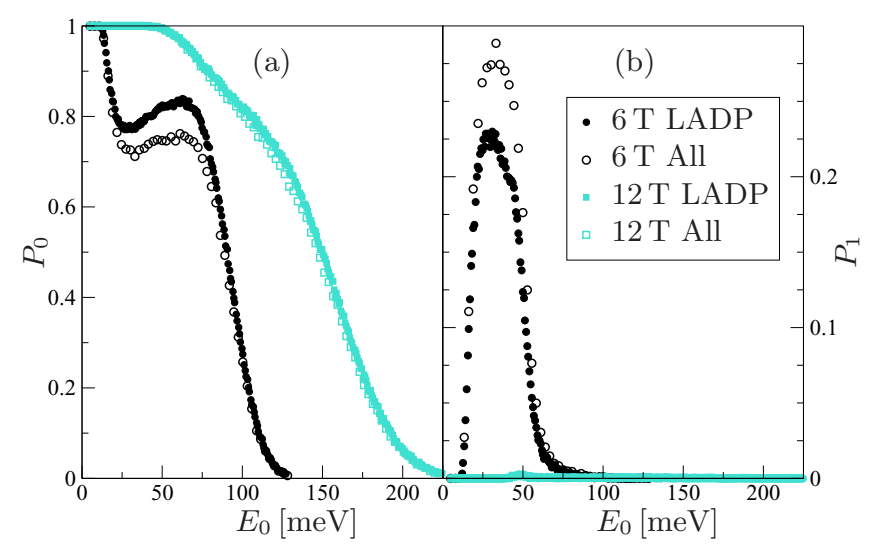

FIG. 8. Survival probabilities (a) $P_{0}$ and (b) $P_{1}$ as a function of injection energy $E_{0}$ for two magnetic field strengths, $B=6,12 \mathrm{~T}$. Solid symbols show results with LO + LADP interactions only. Open symbols show results with LO + all three acoustic phonon interactions. 
[1] M. D. Blumenthal, B. Kaestner, L. Li, S. Giblin, T. J. B. M. Janssen, M. Pepper, D. Anderson, G. Jones, and D. A. Ritchie, Nat. Phys. 3, 343 (2007).

[2] B. Kaestner, V. Kashcheyevs, S. Amakawa, M. D. Blumenthal, L. Li, T. J. B. M. Janssen, G. Hein, K. Pierz, T. Weimann, U. Siegner, and H. W. Schumacher, Phys. Rev. B 77, 153301 (2008).

[3] C. Leicht, P. Mirovsky, B. Kaestner, F. Hohls, V. Kashcheyevs, E. V. Kurganova, U. Zeitler, T. Weimann, K. Pierz, and H. W. Schumacher, Semicond. Sci. Technol. 26, 055010 (2011).

[4] M. Kataoka, J. D. Fletcher, P. See, S. P. Giblin, T. J. B. M. Janssen, J. P. Griffiths, G. A. C. Jones, I. Farrer, and D. A. Ritchie, Phys. Rev. Lett. 106, 126801 (2011).

[5] S. Giblin, M. Kataoka, J. Fletcher, P. See, T. Janssen, J. Griffiths, G. Jones, I. Farrer, and D. Ritchie, Nat. Commun. 3, 930 (2012).

[6] J. D. Fletcher, M. Kataoka, S. P. Giblin, S. Park, H.-S. Sim, P. See, D. A. Ritchie, J. P. Griffiths, G. A. C. Jones, H. E. Beere, and T. J. B. M. Janssen, Phys. Rev. B 86, 155311 (2012).

[7] J. D. Fletcher, P. See, H. Howe, M. Pepper, S. P. Giblin, J. P. Griffiths, G. A. C. Jones, I. Farrer, D. A. Ritchie, T. J. B. M. Janssen, and M. Kataoka, Phys. Rev. Lett. 111, 216807 (2013).

[8] J. Waldie, P. See, V. Kashcheyevs, J. P. Griffiths, I. Farrer, G. A. C. Jones, D. A. Ritchie, T. J. B. M. Janssen, and M. Kataoka, Phys. Rev. B 92, 125305 (2015).

[9] G. Fève, A. Mahé, J.-M. Berroir, T. Kontos, B. Plaçais, D. C. Glattli, A. Cavanna, B. Etienne, and Y. Jin, Science 316, 1169 (2007).

[10] M. Kataoka, M. R. Astley, A. L. Thorn, D. K. L. Oi, C. H. W. Barnes, C. J. B. Ford, D. Anderson, G. A. C. Jones, I. Farrer, D. A. Ritchie, and M. Pepper, Phys. Rev. Lett. 102, 156801 (2009).

[11] R. P. G. McNeil, M. Kataoka, C. J. B. Ford, C. H. W. Barnes, D. Anderson, G. A. C. Jones, I. Farrer, and D. A. Ritchie, Nature (London) 477, 439 (2011).

[12] S. Hermelin, S. Takada, M. Yamamoto, S. Tarucha, A. D. Wieck, L. Saminadayar, C. Bauerle, and T. Meunier, Nature (London) 477, 435 (2011).

[13] J. Dubois, T. Jullien, F. Portier, P. Roche, A. Cavanna, Y. Jin, W. Wegscheider, P. Roulleau, and D. C. Glattli, Nature (London) 502, 659 (2013).

[14] Y. Ji, Y. Chung, D. Sprinzak, M. Heiblum, D. Mahalu, and H. Shtrikman, Nature (London) 422, 415 (2003).

[15] L. V. Litvin, H.-P. Tranitz, W. Wegscheider, and C. Strunk, Phys. Rev. B 75, 033315 (2007).

[16] P. Roulleau, F. Portier, D. C. Glattli, P. Roche, A. Cavanna, G. Faini, U. Gennser, and D. Mailly, Phys. Rev. B 76, 161309 (2007).

[17] P. Roulleau, F. Portier, P. Roche, A. Cavanna, G. Faini, U. Gennser, and D. Mailly, Phys. Rev. Lett. 100, 126802 (2008).

[18] P. Roulleau, F. Portier, P. Roche, A. Cavanna, G. Faini, U. Gennser, and D. Mailly, Phys. Rev. Lett. 101, 186803 (2008).

[19] P. Roulleau, F. Portier, P. Roche, A. Cavanna, G. Faini, U. Gennser, and D. Mailly, Phys. Rev. Lett. 102, 236802 (2009).
[20] E. Bieri, M. Weiss, O. Göktas, M. Hauser, C. Schönenberger, and S. Oberholzer, Phys. Rev. B 79, 245324 (2009).

[21] E. Bocquillon, F. D. Parmentier, C. Grenier, J.-M. Berroir, P. Degiovanni, D. C. Glattli, B. Plaçais, A. Cavanna, Y. Jin, and G. Fève, Phys. Rev. Lett. 108, 196803 (2012).

[22] N. Ubbelohde, F. Hohls, V. Kashcheyevs, T. Wagner, L. Fricke, B. Kästner, K. Pierz, H. W. Schumacher, and R. J. Haug, Nat. Nano 10, 46 (2015).

[23] V. Freulon, A. Marguerite, J.-M. Berroir, B. Placais, A. Cavanna, Y. Jin, and G. Feve, Nat. Commun. 6, 6854 (2015).

[24] S. Tewari, P. Roulleau, C. Grenier, F. Portier, A. Cavanna, U. Gennser, D. Mailly, and P. Roche, Phys. Rev. B 93, 035420 (2016).

[25] D. Taubert, C. Tomaras, G. J. Schinner, H. P. Tranitz, W. Wegscheider, S. Kehrein, and S. Ludwig, Phys. Rev. B 83, 235404 (2011).

[26] N. Johnson, C. Emary, S. Ryu, H.-S. Sim, P. See, J. D. Fletcher, J. P. Griffiths, G. A. C. Jones, I. Farrer, D. A. Ritchie, M. Pepper, T. J. B. M. Janssen, and M. Kataoka, Phys. Rev. Lett. 121, 137703 (2018).

[27] C. Emary, A. Dyson, S. Ryu, H.-S. Sim, and M. Kataoka, Phys. Rev. B 93, 035436 (2016).

[28] U. Bockelmann and G. Bastard, Phys. Rev. B 42, 8947 (1990).

[29] S. Komiyama, H. Hirai, M. Ohsawa, Y. Matsuda, S. Sasa, and T. Fujii, Phys. Rev. B 45, 11085 (1992).

[30] W. Xu, Phys. Rev. B 54, 2775 (1996).

[31] S. Datta, Electronic Transport in Mesoscopic Systems, Cambridge Studies in Semiconductor Physics and Microelectronic Engineering No. 3 (Cambridge University Press, Cambridge, UK, 1997).

[32] M. Kataoka, N. Johnson, C. Emary, P. See, J. P. Griffiths, G. A. C. Jones, I. Farrer, D. A. Ritchie, M. Pepper, and T. J. B. M. Janssen, Phys. Rev. Lett. 116, 126803 (2016).

[33] H. Fröhlich, Adv. Phys. 3, 325 (1954).

[34] G. D. Mahan, Many-Particle Physics, 3rd ed. (Springer, New York, 2000).

[35] J. I. Climente, A. Bertoni, G. Goldoni, and E. Molinari, Phys. Rev. B 74, 035313 (2006).

[36] V. Gantmakher and Y. Levinson, Carrier Scattering in Metals and Semiconductors (Elsevier, New York, 1987).

[37] H.-P. Breuer and F. Petruccione, The Theory of Open Quantum Systems (Oxford University Press, Oxford, UK, 2007).

[38] B. K. Ridley, Electrons and Phonons in Semiconductor Multilayers, 2nd ed. (Cambridge University Press, Cambridge, UK, 2009).

[39] G. Allcock, Ann. Phys. 53, 253 (1969).

[40] R. S. Dumont and T. L. Marchioro II, Phys. Rev. A 47, 85 (1993).

[41] V. Delgado, Phys. Rev. A 59, 1010 (1999).

[42] J. Muga and C. Leavens, Phys. Rep. 338, 353 (2000).

[43] M. Kataoka, J. D. Fletcher, and N. Johnson, Phys. Status Solidi B 254, 1600547 (2017).

[44] S. Ryu, M. Kataoka, and H.-S. Sim, Phys. Rev. Lett. 117, 146802 (2016).

[45] N. Johnson, J. D. Fletcher, D. A. Humphreys, P. See, J. P. Griffiths, G. A. C. Jones, I. Farrer, D. A. Ritchie, M. Pepper, 
T. J. B. M. Janssen, and M. Kataoka, Appl. Phys. Lett. 110, 102105 (2017).

[46] H. Risken and T. Frank, The Fokker-Planck Equation: Methods of Solution and Applications (Springer, New York, 1996).

[47] P. Degiovanni, C. Grenier, and G. Fève, Phys. Rev. B 80, 241307 (2009).
[48] D. Ferraro, B. Roussel, C. Cabart, E. Thibierge, G. Fève, C. Grenier, and P. Degiovanni, Phys. Rev. Lett. 113, 166403 (2014).

[49] C. Wahl, J. Rech, T. Jonckheere, and T. Martin, Phys. Rev. Lett. 112, 046802 (2014).

[50] T. Ando, A. B. Fowler, and F. Stern, Rev. Mod. Phys. 54, 437 (1982). 